\title{
LMI-based $\mathrm{H}_{2}$ Control for T-S Fuzzy System with Hard Constraints
}

\author{
Gao Xingquan ${ }^{\mathrm{a}^{*}}$ and Guo Shuang ${ }^{\mathrm{b}}$ \\ ${ }^{a}$ College of information and control, Jilin University of Chemical Technology \\ ${ }^{b}$ Jilin Institute of Science and Technology Information \\ *xqgao_jl@126.com
}

\begin{abstract}
For T-S fuzzy systems with hard constraints and disturbances modeled by impulses, a constrained $\mathrm{H}_{2}$ control scheme in the framework of LMI (Linear Matrix Inequality) optimization is proposed in this paper. First, the $\mathrm{H}_{2}$ norm is chosen as measurement of disturbance attenuation level, and sufficient conditions for guaranteeing an $\mathrm{H}_{2}$ performance are given. Then, under the bound assumption of the disturbance energy, another fixed ellipsoid containing all perturbed trajectories is confirmed. Finally, hard constraints are enforced and translated into LMI conditions. The multi-objective design is lead to solving an optimization problem with LMI constraints. Simulation results for application in TORA system are discussed.
\end{abstract}

Keywords: T-S Fuzzy System; $H_{2}$ control; LMI; hard constraints.

\section{Introduction}

Most real world control systems are nonlinear and must deal with hard constraints [1-2], e.g., control inputs are limited due to the saturation nature of the actuator, some states or process outputs must lie between pre-specified ranges arising from safety, stringent product specifications or environmental regulation. In addition, there exist kinds of external disturbances, which may degrade the performance of the control system and even lead to instability in some cases. Hence, disturbance attenuation control problem of nonlinear system with hard constraints is a valuable research issue.

As a convenient and flexible tool for handling complex nonlinear systems, T-S fuzzy model [3] and control of T-S fuzzy systems received considerable interests, with which one can design controller for nonlinear systems using mature linear control theory. A number of results have appeared on disturbance attenuation control synthesis for T-S fuzzy systems, such as $\mathrm{H}_{\infty}$ control [4-14], $\mathrm{H}_{2}$ control [15-16], generalized $\mathrm{H}_{2}$ control [17], $\mathrm{H}_{2} / \mathrm{H}_{\infty}$ control [18-20], etc. It is somewhat unfortunately, the fore-mentioned approaches were developed only focusing on the disturbance attenuation performance, there is no direct way to enforce constraints on the control inputs or outputs. Primary contributions to satisfaction of constraints for T-S fuzzy systems can be found for example in [21], where an invariant state ellipsoid (for all) is employed to enforce the constraints. This implies that the satisfaction guarantee of constraints is obtained under the assumption of no external disturbances imposed on the systems. But in actual applications, the assumption is too restrict and even impossible.

In this paper, we exploit a constrained state-feedback controller design scheme for T-S fuzzy systems which suffer from the disturbances modeled by impulses. Since the $\mathrm{H}_{2}$ norm is the square root of the total output energy in the impulse response of the system [22], so for the impulse disturbance, the $\mathrm{H}_{2}$ norm is more suitable and less conservative as the measurement of disturbance attenuation level than the $\mathrm{H}_{\infty}$ norm. A main aim of this paper is 
to design state feedback controller such that the $\mathrm{H}_{2}$ norm form disturbance to performance output is minimized. First, sufficient conditions for guaranteeing an $\mathrm{H}_{2}$ performance level and the ellipsoid set including the initial state are given. With the assumption of the disturbance energy bound, we seek a fixed ellipsoid set containing all perturbed trajectories, i.e. the state of the closed-loop system is guarantee staying in the set when there exist some disturbances whose energy under a certain bound. Then time-domain hard constraints are enforced and translated into LMI conditions using the concept of reachable sets and state-space ellipsoids. Finally, the multi-objective design is lead to solving an optimization problem with LMI constraints.

This paper is structured as follows: Section 2 describes the control problem of T-S fuzzy systems with hard constraints in the frame work of multi-objective control. In Section 3, a state-feedback solution is derived on LMI optimization. Simulation results for TORA system are given and discussed in Section 4.

\section{Control Problem}

Consider a nonlinear system with external disturbances can be described by the following T-S fuzzy model

$$
\begin{array}{ll}
R^{i}: & \\
\text { IF } & \lambda_{1}(t) \text { is } M_{1}^{i} \text { and } \lambda_{2}(t) \text { is } M_{2}^{i}, \cdots \text { and } \lambda_{r_{1}}(t) \text { is } M_{r_{1}}^{i} \\
\text { THEN } & \dot{\boldsymbol{x}}(t)=\boldsymbol{A}_{i} \boldsymbol{x}(t)+\boldsymbol{B}_{1, i} \boldsymbol{w}(t)+\boldsymbol{B}_{2, i} \boldsymbol{u}(t), \quad x(0)=x_{0}, \\
& \boldsymbol{z}_{1}(t)=\boldsymbol{C}_{1, i} \boldsymbol{x}(t)+\boldsymbol{D}_{1 u, i} \boldsymbol{u}(t), \\
& \boldsymbol{z}_{2}(t)=\boldsymbol{C}_{2, i} \boldsymbol{x}(t)+\boldsymbol{D}_{2 u, i} \boldsymbol{u}(t), i=1,2 \cdots, r,
\end{array}
$$

subject to hard constrains (including control constraints, state constraints or output constraints)

$$
\left|z_{2 v}(t)\right| \leq z_{2 v, \max }, \quad v=1,2, \cdots, n_{z 2}, \quad t \geq 0,
$$

where $\boldsymbol{R}^{i}$ denotes rule $i, M_{j}^{i}$ is fuzzy set and $r$ is the number of model rule, $\lambda(\boldsymbol{t}) \in \boldsymbol{R}^{r_{1}}$ is the known premise variable which can be measured directly or be function of the measurable variable, and/or time, $\boldsymbol{x}(\boldsymbol{t}) \in \boldsymbol{R}^{n}$ is the state, $\boldsymbol{u}(\boldsymbol{t}) \in \boldsymbol{R}^{m_{u}}$ is the control input, $\boldsymbol{w}(\boldsymbol{t}) \in \boldsymbol{R}^{\boldsymbol{m}_{w}}$ is the external disturbances, $z_{1}(\boldsymbol{t}) \in \boldsymbol{R}^{z_{1}}$ is the performance output, $z_{2}(\boldsymbol{t}) \in \boldsymbol{R}^{n_{22}}$ is the constrained output.

For the T-S fuzzy system (1), assume $C^{i}$ is the state-feedback controller designed for the $i$ th subsystem

$$
\begin{array}{lc}
C^{i}: \text { IF } & \lambda_{1}(t) \text { is } M_{1}^{i} \text { and } \lambda_{2}(t) \text { is } M_{2}^{i}, \cdots \text { and } \lambda_{r_{1}}(t) \text { is } M_{r_{1}}^{i} \\
\text { THEN } & \boldsymbol{u}(t)=\boldsymbol{K}_{i} \boldsymbol{x}(t), \quad i=1,2, \cdots, r,
\end{array}
$$

here $\boldsymbol{K}_{i} \in \mathbf{R}^{m_{u} \times n}$ is a constant feedback gain to be determined. Via PDC[23], the overall fuzzy controller is constituted by

$$
\boldsymbol{u}(t)=\sum_{i=1}^{r} h_{i}(\lambda) \boldsymbol{K}_{i} \boldsymbol{x}(t)
$$

where $h_{i}$ is weighted coefficient of the $i$ th sub-controller in the whole controller. Its definition and calculation please refer to relevant literatures, and the same below. 
The disturbance attenuation control problem for T-S fuzzy system with hard constraints discussed in this paper can formulated in the framework of multi-objective control as follows:

Find a controller (4) such that, the closed-loop system is internally stable, the $\mathrm{H}_{2}$ norm from the disturbance $w$ to the controlled output $z_{1}$ is minimized, while the hard constraints (2) are satisfied.

\section{Constrained ${ }_{2}$ Control for T-S Fuzzy System}

This section describes a state feedback solution to the above control problem based on LMI optimization. Using state feedback (4), the closed-loop system is then be described by the parameter-varying linear system

$$
\begin{aligned}
& \dot{\boldsymbol{x}}(t)=\boldsymbol{A}_{c l}(\lambda) \boldsymbol{x}(t)+\boldsymbol{B}_{1, c l}(\lambda) \boldsymbol{w}(t), \boldsymbol{x}(0)=x_{0}, \\
& \boldsymbol{z}_{1}(t)=\boldsymbol{C}_{1, c l}(\lambda) \boldsymbol{x}(t), \\
& \boldsymbol{z}_{2}(t)=\boldsymbol{C}_{2, c l}(\lambda) x(t)
\end{aligned}
$$

with $\quad \boldsymbol{A}_{c l}(\lambda)=\sum_{i=1}^{r} \sum_{j=1}^{r} \boldsymbol{h}_{i}(\lambda) \boldsymbol{h}_{j}(\lambda)\left\{\boldsymbol{A}_{i}+\boldsymbol{B}_{2, i} \boldsymbol{K}_{j}\right\}, \quad \boldsymbol{C}_{1, c l}(\lambda)=\sum_{i=1}^{r} \sum_{j=1}^{r} \boldsymbol{h}_{i}(\lambda) \boldsymbol{h}_{j}(\lambda)\left\{\boldsymbol{C}_{1, i}+\boldsymbol{D}_{1 u, i} \boldsymbol{K}_{j}\right\}$, $\boldsymbol{B}_{1, c l}(\lambda)=\sum_{i=1}^{r} \boldsymbol{h}_{i}(\lambda) \boldsymbol{B}_{1, i}, \boldsymbol{C}_{2, c l}(\lambda)=\sum_{i=1}^{r} \sum_{j=1}^{r} h_{i}(\lambda) h_{j}(\lambda)\left\{\boldsymbol{C}_{2, i}+\boldsymbol{D}_{2 u, i} \boldsymbol{K}_{j}\right\}$.

In the framework of multi-objective control, it is shown that $\boldsymbol{A}_{c l}(\lambda)$ is stable, the $\mathrm{H}_{2}$ norm from the disturbance $w$ to the controlled output $z_{1}$ less than $\gamma$, if and only if there exist $\boldsymbol{P}=\boldsymbol{P}^{T}>0$ and $\boldsymbol{S}>0$ such that[24]

$$
\begin{gathered}
{\left[\begin{array}{cc}
\boldsymbol{A}_{c l}^{T}(\lambda) \boldsymbol{P}+\boldsymbol{P} \boldsymbol{A}_{c l}(\lambda) & \boldsymbol{P} \boldsymbol{B}_{1, c l}(\lambda) \\
\boldsymbol{B}_{1, c l}^{T}(\lambda) \boldsymbol{P} & -\boldsymbol{I}
\end{array}\right]<0,} \\
{\left[\begin{array}{cc}
\boldsymbol{P} & \boldsymbol{C}_{1, c l}^{T}(\lambda) \\
\boldsymbol{C}_{1, c l}(\lambda) & \boldsymbol{S}
\end{array}\right]>0, \quad \operatorname{Tr}(\boldsymbol{S})<\gamma .}
\end{gathered}
$$

In the following, we seek conditions for guaranteeing the satisfaction of constraints. With a candidate Lyapunov function $V(\boldsymbol{x})=\boldsymbol{x}^{T} \boldsymbol{P} \boldsymbol{x}$, it is easy to prove that (6) implies

$$
\frac{d}{d t} V(\boldsymbol{x}(t))-\boldsymbol{w}^{T}(t) \boldsymbol{w}(t)<0
$$

The integration of both sides from 0 to $t$ then yields

$$
V(\boldsymbol{x}(t))-V(\boldsymbol{x}(0))<\int_{0}^{t} \boldsymbol{w}^{T}(\tau) \boldsymbol{w}(\tau) d \tau .
$$

Assume the disturbances energy is bounded, i.e. $\int_{0}^{\infty} \boldsymbol{w}^{T}(\tau) \boldsymbol{w}(\tau) d \tau \leq w_{\max }$. Then, (7) leads to

$$
V(\boldsymbol{x}(t))<V(\boldsymbol{x}(0))+\int_{0}^{t} \boldsymbol{w}^{T}(\tau) \boldsymbol{w}(\tau) d \tau<V(\boldsymbol{x}(0))+w_{\max } .
$$

With the definition $\alpha:=V(\boldsymbol{x}(0))+w_{\max },(8)$ implies that the closed-loop state trajectory stays in an ellipsoid defined by

$$
\Omega(\boldsymbol{P}, \alpha):=\left\{\boldsymbol{x} \in R^{n} \mid V(\boldsymbol{x})<\alpha\right\} .
$$


In other words, the fixed ellipsoid set $\Omega(\boldsymbol{P}, \alpha)$ contains all possible perturbed trajectories with disturbances whose energy is bounded by $\alpha(\boldsymbol{x}(0)=0$ is assumed). With the ellipsoid set, we can cast the hard constraints into matrix inequalities.

Introduce $\boldsymbol{e}_{v}$ as the standard basis vector in constrained output space, then $v$ th constrained output can be rewritten as $\boldsymbol{z}_{2 v}(t)=\boldsymbol{e}_{v}^{T} \boldsymbol{C}_{2, c l}(\lambda) \boldsymbol{x}(t)$ and the corresponding hard constraint of (2) is equivalent to

$$
\boldsymbol{x}^{T}(t) \frac{\boldsymbol{C}_{2, c l}^{T}(\lambda) \boldsymbol{e}_{v} \boldsymbol{e}_{v}^{T} \boldsymbol{C}_{2, c l}(\lambda)}{z_{2 v, \max }^{2}} \boldsymbol{x}(t) \leq 1,
$$

Since $V(\boldsymbol{x}(t))=\boldsymbol{x}^{T}(t) \boldsymbol{P x}(t)<\alpha$ for $t>0$, if

$$
\boldsymbol{x}^{T}(t) \frac{\boldsymbol{C}_{2, c l}^{T}(\boldsymbol{\lambda}) \boldsymbol{e}_{v} \boldsymbol{e}_{v}^{T} \boldsymbol{C}_{2, c l}(\boldsymbol{\lambda})}{\boldsymbol{z}_{2 v, \max }^{2}} \boldsymbol{x}(t) \leq \frac{V(\boldsymbol{x}(t))}{\alpha},
$$

then (10) holds. It is equivalent to

$$
\boldsymbol{x}^{T}(t)\left(\frac{\boldsymbol{C}_{2, c l}^{T}(\lambda) \boldsymbol{e}_{\boldsymbol{v}} \boldsymbol{e}_{v}^{T} \boldsymbol{C}_{2, c l}(\lambda)}{z_{2 v, \max }^{2}}-\frac{\boldsymbol{P}}{\alpha}\right) \boldsymbol{x}(t) \leq 0,
$$

for any $\boldsymbol{x}(t) \neq 0$, if

$$
\frac{\boldsymbol{P}}{\alpha}-\frac{\boldsymbol{C}_{2, c l}^{T}(\lambda) \boldsymbol{e}_{v} \boldsymbol{e}_{v}^{T} \boldsymbol{C}_{2, c l}(\lambda)}{\boldsymbol{z}_{2 v, \max }^{2}} \geq 0,
$$

(11) hold, in other word, the constraints are satisfied. By Schur complement, we arrive at the equivalence of the above inequality

$$
\left[\begin{array}{cc}
\frac{z_{2 v, \text { max }}^{2}}{\alpha} & \boldsymbol{e}_{v}^{T} \boldsymbol{C}_{2, c l}(\lambda) \\
\boldsymbol{C}_{2, c l}^{T}(\lambda) \boldsymbol{e}_{v} & \boldsymbol{P}
\end{array}\right] \geq 0 .
$$

The above discussion can be summarized as follows.

Lemma1 For a given positive scalar $\gamma>0$, if there exist symmetric $\boldsymbol{P}>0$ satisfying (6a), (6b) and (14), then, the system (5) is stable and the $H_{2}$ norm from the disturbance $w$ to the controlled output $z_{1}$ less than $\gamma$.

Substitute $\boldsymbol{A}_{c l}(\lambda), \quad \boldsymbol{B}_{1, c l}(\lambda), \boldsymbol{C}_{1, c l}(\lambda)$ and $\boldsymbol{C}_{2, c l} \quad(\lambda)$ into (6) and (14). By defining $\boldsymbol{Q}=\boldsymbol{P}^{-1}, \boldsymbol{Y}_{i}=\boldsymbol{K}_{i} \boldsymbol{Q}$, pre- and post-multiplying both sides of (6) with $\operatorname{diag}(\boldsymbol{Q}, \boldsymbol{I})$, and pre- and post-multiplying both sides of (14) with $\operatorname{diag}(\boldsymbol{I}, \boldsymbol{Q}),(6)$ and (14) become

$$
\begin{gathered}
\sum_{i=1}^{r} \sum_{j=1}^{r} \boldsymbol{h}_{i}(\lambda) \boldsymbol{h}_{j}(\lambda) \boldsymbol{M}_{i j}<0, \\
\sum_{i=1}^{r} \sum_{j=1}^{r} \boldsymbol{h}_{i}(\lambda) \boldsymbol{h}_{j}(\lambda)<0, \operatorname{Tr}(\boldsymbol{S})<\gamma, \\
\sum_{i=1}^{r} \sum_{j=1}^{r} \boldsymbol{h}_{i}(\lambda) \boldsymbol{h}_{j}(\lambda) \boldsymbol{H}_{i j . v} \geq 0,
\end{gathered}
$$




$$
\begin{aligned}
& \text { Here } \quad \boldsymbol{M}_{i j}=\left[\begin{array}{cc}
\boldsymbol{Q} \boldsymbol{A}_{i}^{T}+\boldsymbol{A}_{i} \boldsymbol{Q}+\boldsymbol{Y}_{j}^{T} \boldsymbol{B}_{2, i}^{T}+\boldsymbol{B}_{2, i} \boldsymbol{Y}_{j} & * \\
\boldsymbol{B}_{1, i}^{T} & -\boldsymbol{I}
\end{array}\right] \quad, \quad \boldsymbol{N}_{i j}=\left[\begin{array}{cc}
\boldsymbol{Q} & * \\
\boldsymbol{C}_{1 i} \boldsymbol{Q}+\boldsymbol{D}_{1 u, i} \boldsymbol{Y}_{j} & \boldsymbol{S}
\end{array}\right], \text {, } \\
& \boldsymbol{H}_{i j, v}=\left[\begin{array}{cc}
\frac{z_{2 v, \max }^{2}}{\alpha} & \boldsymbol{e}_{v}^{T}\left(\boldsymbol{C}_{2, i} \boldsymbol{Q}+\boldsymbol{D}_{2 u, i} \boldsymbol{Y}_{j}\right) \\
\left(\boldsymbol{C}_{2, i} \boldsymbol{Q}+\boldsymbol{D}_{2 u, i} \boldsymbol{Y}_{j}\right) \boldsymbol{e}_{v} & \boldsymbol{Q}
\end{array}\right] \text {. }
\end{aligned}
$$

Note (15)-(17) are parameter-varying matrix inequalities. From [25], one can infer that the following LMIs are sufficient conditions of MIs (15)-(17).

$$
\begin{gathered}
\boldsymbol{M}_{i i} \leq 0, \frac{1}{r-1} \boldsymbol{M}_{i i}+\frac{1}{2}\left(\boldsymbol{M}_{i j}+\boldsymbol{M}_{j i}\right) \leq 0, \\
\boldsymbol{N}_{i i}>0, \frac{1}{r-1} \boldsymbol{N}_{i i}+\frac{1}{2}\left(\boldsymbol{N}_{i j}+\boldsymbol{N}_{j i}\right)>0, \quad \operatorname{Tr}(\boldsymbol{S})<\gamma \\
\boldsymbol{H}_{i i, v}>0, \frac{1}{r-1} \boldsymbol{H}_{i i, v}+\frac{1}{2}\left(\boldsymbol{H}_{i j, v}+\boldsymbol{H}_{j i, v}\right)>0, \\
\quad i, j=1,2, \cdots r, \quad i \neq j, v=1,2, \cdots, n_{z 2} .
\end{gathered}
$$

which are LMIs in $\gamma, \boldsymbol{Q}, \boldsymbol{Y}_{i}$ and $S$ for a fixed $\alpha$.Then the following optimization problem

$$
\min _{\gamma, \boldsymbol{Q}, S, \boldsymbol{Y}_{j}(j=1,2, \cdots r)} \gamma \quad \text { s.t. LMIs (18)-(20) }
$$

is then convex and numerically tractable.

We are now at the place to state the following result.

Theorem 1 If there exists an ( almost) optimal solution $\left(\gamma^{*}, \boldsymbol{Q}^{*}, \boldsymbol{Y}_{i}^{*}, S^{*}\right)$ to optimization problem (21), the $i$ th state feedback gain can be computed by $\boldsymbol{K}_{i}=\boldsymbol{Y}_{i}^{*} \boldsymbol{Q}^{*-1}$ and the closedloop system (5) with controller (4) achieves :

I. that it is globally asymptotically stable;

II. a $\mathrm{H}_{2}$ disturbance attenuation level $\gamma^{*}$ for external disturbances;

III. that hard constraints are guaranteed to be satisfied for all disturbances whose energy is below $\alpha(\boldsymbol{x}(0)=0$ is assumed).

Remark 1 As an adjustable parameter for controller, the value of $\alpha$ reflects the trade-off between achieving good performance and respecting hard constraints, so it can be chosen according to the priory knowledge about the disturbances and/or an expected disturbance attenuation level. If there probably exist large disturbances or we hope the close-loop system keeping high attenuation level for large disturbances, one should choose a large $\alpha$, which probably leads to low performance. Otherwise, enforcing high performance levels (small $\alpha$ ) requires to reduce $\alpha$, which might result in control constraints violation in case that the system is affected by unexpectedly large disturbances. So a priori knowledge on disturbances is very useful in determining a suitable $\alpha$ 


\section{Simulation Application: TORA System}

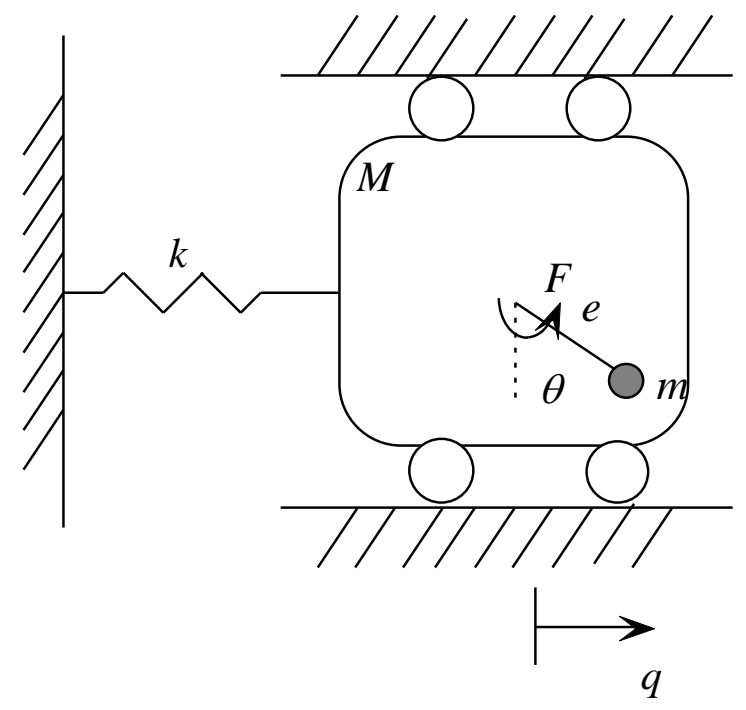

Figure 1. Diagrammatic Sketch of TORA

Consider a TORA system (Translational oscillator with an eccentric rotational proof mass actuator, see Figure 1) can be described by the following T-S fuzzy model [26]:

$$
\begin{gathered}
A_{1}=\left[\begin{array}{cccc}
0 & 1 & 0 & 0 \\
-1 & 0 & \varepsilon \frac{\sin \beta \pi}{\beta \pi} & 0 \\
0 & 0 & 0 & 1 \\
\frac{-\varepsilon}{1-\varepsilon^{2}} & 0 & 0 & 0
\end{array}\right], \boldsymbol{A}_{2}=\left[\begin{array}{cccc}
0 & 1 & 0 & 0 \\
-1 & 0 & \varepsilon \frac{2}{\pi} & 0 \\
0 & 0 & 0 & 1 \\
0 & 0 & 0 & 0
\end{array}\right], \boldsymbol{A}_{3}=\left[\begin{array}{cccc}
0 & 1 & 0 & 0 \\
-1 & 0 & \varepsilon & 0 \\
0 & 0 & 0 & 1 \\
\frac{\varepsilon}{1-\varepsilon^{2}} & 0 & \frac{-\varepsilon^{2}}{1-\varepsilon^{2}} & 0
\end{array}\right], \\
\boldsymbol{A}_{4}=\left[\begin{array}{cccc}
0 & 1 & 0 & 0 \\
-1 & 0 & \varepsilon & 0 \\
0 & 0 & 0 & 1 \\
\frac{\varepsilon}{1-\varepsilon^{2}} & 0 & \frac{-\varepsilon^{2}\left(1+a^{2}\right)}{1-\varepsilon^{2}} & 0
\end{array}\right], \boldsymbol{B}_{2,1}=\left[\begin{array}{c}
0 \\
0 \\
0 \\
1 \\
0 \\
1-\varepsilon^{2}
\end{array}\right], \boldsymbol{B}_{2,2}=\left[\begin{array}{c}
0 \\
0 \\
0 \\
1
\end{array}\right], \boldsymbol{B}_{2,3}=\left[\begin{array}{c}
0 \\
0 \\
1 \\
\frac{1-\varepsilon^{2}}{0}
\end{array}\right], \\
\boldsymbol{B}_{2,4}=\left[\begin{array}{c}
0 \\
\frac{1}{1-\varepsilon^{2}}
\end{array}\right], \beta=0.99, \varepsilon=0.1, a=4, \boldsymbol{B}_{1, i}=\boldsymbol{B}_{2, i}(i=1,2, \cdots 4) .
\end{gathered}
$$

Because of actuator saturation and mechanical structure constraints, there exist the following hard constraints for this system

$$
|u| \leq 1=u_{\max },\left|x_{4}\right| \leq 2=y_{\max } .
$$


A control problem of this system is to design a controller such that the closed-loop system has a certain disturbance attenuation level while the hard constraints are respected. When solving the controller gains, we choose the same controlled output parameters, that is

$$
\boldsymbol{C}_{1, i}=\left[\begin{array}{cccc}
10 & 0 & 0 & 0 \\
0 & 1 & 0 & 0 \\
0 & 0 & 1 & 0 \\
0 & 0 & 0 & 0.1
\end{array}\right], \boldsymbol{D}_{1 u, i}=\left[\begin{array}{l}
0 \\
0 \\
0 \\
0
\end{array}\right], \quad i=1,2, \cdots, 4 .
$$

From (9), the constrained output matrix selected is

$$
\boldsymbol{C}_{2, i}=\left[\begin{array}{llll}
0 & 0 & 0 & 1 \\
0 & 0 & 0 & 0
\end{array}\right], \boldsymbol{D}_{2 u, i}=\left[\begin{array}{l}
0 \\
1
\end{array}\right], i=1,2, \cdots, 4, z_{\max }=\left[\begin{array}{l}
u_{\max } \\
y_{\max }
\end{array}\right] .
$$

Parameter $\alpha$ can be treated as adjustable controller parameter, Figure 2. shows the relationship of the $\mathrm{H}_{2}$ performance level $\gamma$ and parameter $\alpha(\boldsymbol{x}(0)=0)$ by solving the optimization problem (21), from which, we can see the increase in $\alpha$ from the value 0.05 to 1.5 results in an increase in $\gamma$ from 0.0166 to 23.44(implies the decrease of control performance). Just as the discussion in Section 3, hoping the system a certain disturbance attenuation level for larger energy disturbances implies a larger $\alpha$ being chosen, but in this case, the control performance will decrease largely.

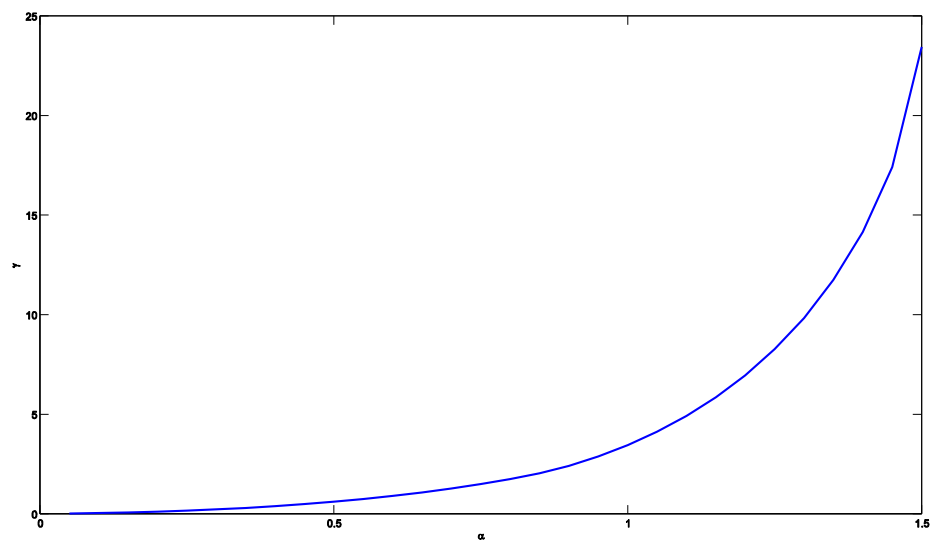

Figure 2. The Effect of Varying Controller Parameter $\alpha(x(0)=0)$ for Performance Level $\gamma$

By solving optimization problem (21), we can obtain T-S fuzzy controllers with different value of $\alpha=0.5,0.8,1$. With these controllers, Figure 3 and Figure 4 show the system responses while imposing an impulse variation in $w$ with a width of $0.05 \mathrm{~s}$, amplitude of 5 at time $t=0$, and the initial state is $x(0)=\left[\begin{array}{llll}0 & 0 & 0 & 0\end{array}\right]^{T}$. From the results we can see in these three cases, the systems all diverge from equilibrium point when the disturbance occurred, and converge to original point (see Fig. 3) when the disturbance disappeared, at the same time, the hard constraints (22) are respected (see Figure 4). We also can see that the value of $\alpha$ is chosen smaller, the control performance is better, but the variables with constraints tend to constraints boundaries more closely (see Figure 4) and the margin of respecting constraints is smaller. Actually, supreme small value of $\alpha$ will lead to violation of the hard constraints. Therefore, the value of $\alpha$ reflects the trade-off between achieving good performance and 
respecting hard constraints, it can be chosen according to the priory knowledge about the disturbances and/or an expected disturbance attenuation level.
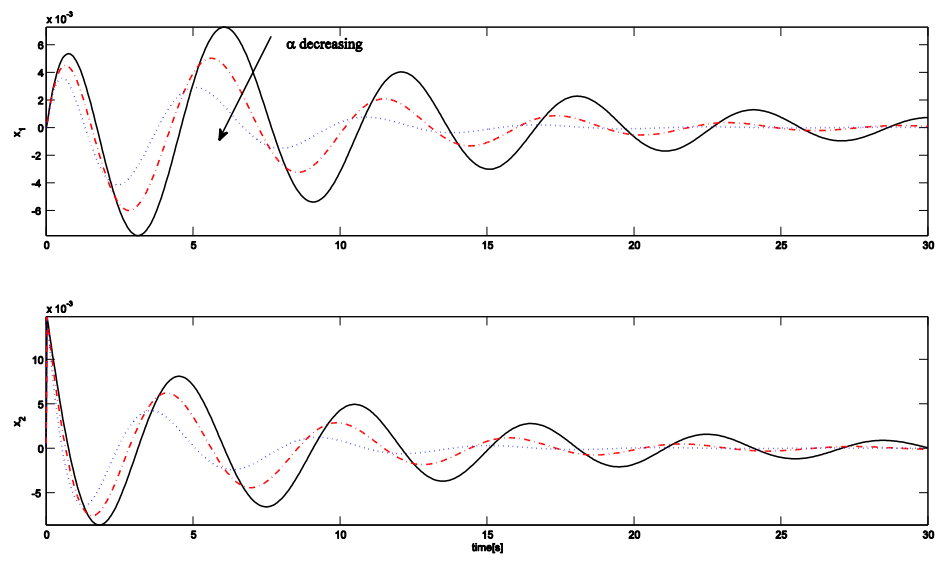

Figure 3. Response ( $x_{1}$ and $\left.x_{2}\right)$ with Different $\alpha(-: \alpha=1,-\cdot-: \alpha=0.75, \cdots \cdots \cdots$ $: \alpha=0.5$ )
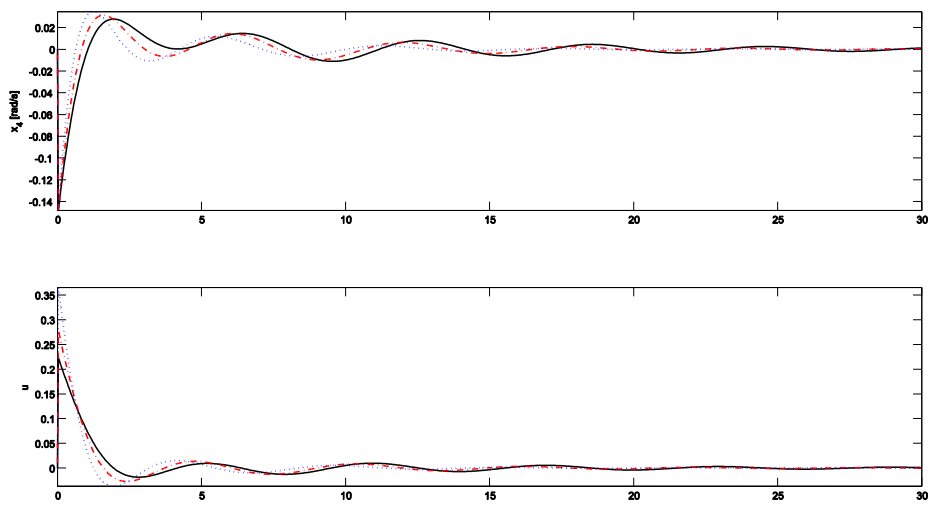

Figure 4. Response $x_{4}$ and Control Input $u$ with Different $\alpha(-: \alpha=1$, $-\cdot-: \alpha=0.75, \cdots \cdots \cdot: \alpha=0.5$ )

\section{Conclusion}

In the framework of multi-objective control, a constrained $\mathrm{H}_{2}$ state-feedback T-S fuzzy control approach is presented. The $\mathrm{H}_{2}$ norm is used as a measurement for disturbance attenuation level, and by the assumption of disturbance energy bound, a fixed ellipsoid set is adapted to translate hard constraints into LMI conditions. The bound value reflects the tradeoff between achieving good performance and respecting hard constraints, so it can be treated as adjustable parameter for controller and can be chosen according to the priory knowledge about the disturbances and/or an expected disturbance attenuation level. A state-feedback solution to the control problem is then given, based on LMI optimization. Simulation results 
for TORA show that the presented approach makes the closed-loop system have a certain disturbance attenuation level with hard constraints are respected.

\section{Acknowledgements}

This work was supported by the National Natural Science Foundation of China (No. 61034001) and the Project of Education Department of Jilin province (20140352).

\section{References}

[1] G. C. Goodwin, M. M. Seron, and J. A. De Doná, "Constrained Control and Estimation: an Optimization Approach", London: Springer (2005).

[2] W. H. T. M. Aangenent, W. P. M. H. Heemels, M. J. G. van de Molengraft, D. Henrion and M. Steinbuch, "Linear control of time-domain constrained systems", Automatica, vol. 48, no. 5, (2012), pp. 736-746.

[3] T. Takagi and M. Sugeno, "Fuzzy identification of systems and its applications to modeling and control", IEEE Trans. Syst. Man. Cyber, vol. 15, no. 1, (1985), pp. 116-132.

[4] K. Tanaka, T. Ikeda and H. O. Wang, "Robust stabilization of a class of uncertain nonlinear systems via fuzzy control: Quadratic stability, Ho control theory, and linear matrix inequalities", IEEE Transactions on Fuzzy Systems, vol. 4, no. 1, (1996), pp. 1-13.

[5] X D. Liu and Q L. Zhang, "Approaches to quadratic stability conditions and $\mathrm{H}_{\infty}$ control designs for T-S fuzzy systems”. IEEE Transactions on Fuzzy Systems, vol. 11, no. 6, (2003), pp. 830-839.

[6] C. Lin, Q. G. Wang and T. H. Lee, "Improvement on observer-based $\mathrm{H}_{\infty}$ control for T-S fuzzy systems", Automatica, vol. 41, (2005), pp. 1651-1656.

[7] J. Dong, Y. Wang and G. Yang, " $\mathrm{H}_{\infty}$ and mixed $\mathrm{H}_{2} / \mathrm{H}_{\infty}$ control of discrete-time $\mathrm{T}-\mathrm{S}$ fuzzy systems with local nonlinear models", Fuzzy Sets and Systems, vol. 164, no. 1, (2011), pp. 1-24.

[8] H. Wang, L. Peng, H. Ju and Y. Wang, " $\mathrm{H}_{\infty}$ state feedback controller design for continuous-time T-S fuzzy systems in finite frequency domain", Information Sciences, vol. 223, no. 20, (2013), pp. 221-235.

[9] P. Wang, S. Yu and H. Chen, "T-S model-based nonlinear moving-horizon $\mathrm{H}_{\infty}$ control and applications", Fuzzy Sets and Systems, vol. 212, no. 1, (2013), pp. 78-96.

[10] C. Peng, Q. L. Han, D. Yue and E. Tian, "Sampled-data robust $\mathrm{H}_{\infty}$ control for T-S fuzzy systems with time delay and uncertainties", Fuzzy Sets and Systems, vol. 179, no. 2, (2011), pp. 20-33.

[11] J. Liu, J. Zhang and Y. Zheng, M. He, "Robust $\mathrm{H}_{\infty}$ control for discrete-time T-S fuzzy systems with input delay", Journal of Control Theory and Applications, vol. 9, no. 2, (2011), pp. 189-194.

[12] M. H. Asemani and V. J. Majd, "A robust observer-based $\mathrm{H}_{\infty}$ controller design for uncertain T-S fuzzy systems with unknown premise variables via LMI”, Fuzzy Sets and Systems, vol. 212, (2013), pp. 21-40.

[13] S. Zhou, G. Feng, J. Lam and S. Xu, "Robust $\mathrm{H}_{\infty}$ control for discrete-time fuzzy systems via basis-dependent lyapunov functions", Information Sciences, vol. 174, (2005), pp. 197-217.

[14] S. Kau, H. Lee, C. M. Yang, C. H. Lee, H. Lin and C. H. Fang, "Robust $\mathrm{H}_{\infty}$ fuzzy static output feedback control of T-S fuzzy systems with parametric uncertainties", Fuzzy Sets and Systems, vol. 158, no. 2, (2007), pp. 135-146.

[15] H. N. Wu, B. S. Chen, C. S. Tseng and H. J. Uang, "Robust $\mathrm{H}_{2}$ fuzzy output feedback control for discretetime nonlinear systems with parametric uncertainties", International Journal of Approximate Reasoning, vol. 46, no. 1, (2007), pp.151-165.

[16] H. N. Wu and K. Y. Cai, " $\mathrm{H}_{2}$ guaranteed cost fuzzy control design for discrete-time nonlinear systems with parameter uncertainty", Automatica, vol. 42, no. 7, (2006), pp. 1183-1188.

[17] L. Wang, G. Feng and T. Hesketh, "Piecewise generalized $\mathrm{H}_{2}$ controller synthesis of discrete-time fuzzy systems", IEE Proceeding on Control Theory and Application, vol. 151, no. 5, (2004), pp. 554-560

[18] Hardiansyah and Junaidi, "Multiobjective $\mathrm{H}_{2} / \mathrm{H}_{\infty}$ Control Design with Regional Pole Constraints", Telkomnika, vol. 10, no. 1, (2012), pp. 103-108.

[19] E. S. Tognetti, R. C. Oliveira, and P. L. D. Peres, "Selective $\mathrm{H}_{2}$ and $\mathrm{H}_{\infty}$ Stabilization of Takagi-Sugeno Fuzzy Systems", IEEE Transactions on Fuzzy Systems, vol. 19, no. 5, (2011), pp. 890-900.

[20] H. N. Wu and K. Y. Cai, "Mixed $\mathrm{H}_{2} / \mathrm{H}_{\infty}$ fuzzy output feedback control design for nonlinear dynamic systems: an LMI approach”, IEEE Trans. Fuzzy Syst., 2000,vol. 8, no. 3, (2000), pp. 249-265.

[21] K. Tanaka, T. Ikeda and H. O. Wang, "Fuzzy regulators and fuzzy observers: relaxed stability conditions and LMI-based designs", IEEE Transactions on Fuzzy Systems, 1998, vol. 6, no. 2, (1998), pp. 250-265.

[22] J. B. Burl, "Linear Optimal Control: $\mathrm{H}_{2}$ and $\mathrm{H}_{\infty}$ Methods", Addison: Wesley, (1998).

[23] M. Sugeno and G. T. Kang. "Fuzzy modeling and control of multilayer incinerator", Fuzzy Sets Syst., vol. 18, no. 3, (1986), pp. 329-346. 
International Journal of Control and Automation

Vol.8, No.3 (2015)

[24] C. Scherer, P. Gahinet and M. Chilali, "Multi-objective Output-Feedback Control via LMI Optimization", IEEE Transactions on Automatic Control, vol. 42, no. 7, (1997), pp. 896-911.

[25] H. D. Tuan, P. Apkarian, T. Narikiyo and Y. Yamamoto, "Parameterized linear matrix inequlity techniques in fuzzy control system design", IEEE Tran. on Fuzzy Systems, vol. 9, no. 2, (2001), pp. 324-332.

[26] K. Tanaka and H. O. Wang. "Fuzzy Control Systems Design and Analysis: an Linear Matrix Inequality Approach”, New York, NY: John Wiley Sons, Inc., (2002). 